\title{
Riesgo cardiovascular en la mujer diabética
}

\section{Cardiovascular risk in the diabetic woman}

\author{
Dora I. Molina de Salazar ${ }^{1,2,3 *}$ y Juan F. Salazar-Espinosa ${ }^{2,4}$ \\ ${ }^{1}$ Departamento de Medicina Interna, Facultad de Ciencias para la Salud, Universidad de Caldas, Manizales; ${ }^{2}$ Centro de Investigación Clínica, IPS \\ Médicos Internistas de Caldas, Manizales; ${ }^{3}$ Asociación Colombiana de centro de investigación clínica-ACIC, Bogotá; ${ }^{4}$ Facultad de Ciencias para \\ la Salud, Universidad de Caldas, Manizales. Colombia
}

\section{Resumen}

Introducción: La enfermedad cardiovascular en mujeres ha sido un apartado con diversas investigaciones que han intentado llegar a los factores de riesgo más determinantes o las vías moleculares más específicas para explicar el riesgo aumentado que poseen las mujeres respecto a los hombres. Objetivo: Dar una visión global de esta situación al lector, involucrando especialmente aquella población de mujeres que padece diabetes mellitus tipo 2, cuya condición es un factor de riesgo independiente para el desarrollo de enfermedad cardiovascular, de gran costo y morbimortalidad mundiales. Métodos: Se realizó una búsqueda en PubMed y Google Scholar con términos MeSH y términos comunes y se obtuvieron algunas referencias cruzadas a criterio de los autores. Conclusiones: Es necesario implementar en la práctica médica diaria un contexto específico de prevención de riesgo cardiovascular mediante programas de educación continuada o por medio de las entidades prestadoras de servicios de salud para evitar la progresión de enfermedad cardiovascular en las mujeres diabéticas antes de llegar a desenlaces mayores y potencialmente fatales.

Palabras clave: Enfermedad cardiovascular. Mujeres. Diabetes mellitus tipo 2.

\section{Abstract}

Introduction: Cardiovascular disease in woman has been an issue with plenty of investigations towards the finding of the most determinant risk factors o the specific molecular paths to explain the increased risk in women compared to men. Objective: To give to the reader a global vision of this situation, involving specially the type 2 diabetes mellitus woman population, whose condition is an independent risk factor to the development of the cardiovascular disease with great cost and morbidity and mortality worldwide. Methods: A PUBMED and Google Scholar search was performed with MeSH and common terms and were obtained some cross-references at the discretion of the authors. Conclusions: It is necessary to implement a specific context of cardiovascular risk prevention in daily medical practice through continuing education programs or through health service providers to prevent the progression of cardiovascular disease in diabetic women before reaching major outcomes and potentially fatal.

Key words: Cardiovascular disease. Women. Diabetes mellitus type 2.

\section{Correspondencia:}

*Dora I. Molina de Salazar E-mail: doraines56@gmail.com
Fecha de recepción: 22-11-2020

Fecha de aceptación: 10-06-2021

DOI: 10.24875/RCCAR.M21000077
Disponible en internet: 29-11-2021 Rev Colomb Cardiol. 2021;28(5):404-409 www.rccardiologia.com 0120-5633 / @ 2021 Sociedad Colombiana de Cardiología y Cirugía Cardiovascular. Publicado por Permanyer. Este es un artículo open access bajo la licencia CC BY-NC-ND (http://creativecommons.org/licenses/by-nc-nd/4.0/). 


\section{Introducción}

La diabetes mellitus tipo II es una enfermedad con aumento mundial progresivo en morbilidad y mortalidad en la población femenina, es un factor de riesgo independiente para cualquier evento cardiovascular mayor $(E C M)$ y aterogénesis ${ }^{1}$. Hay estudios que respaldan el aumento del riesgo relativo para padecer desenlaces cardiovasculares asociados a diabetes mellitus tipo 2 en mujeres comparado con hombres y que el seguimiento de estilos de vida saludables puede disminuir hasta en un $50 \%$ las complicaciones microvasculares y macrovasculares ${ }^{2,3}$. Son indudables las diferencias fisiológicas en el desarrollo de enfermedad cardiovascular entre hombres y mujeres. Se sabe que las mujeres premenopáusicas poseen un factor protector para el desarrollo de eventos cardiovasculares frente a los hombres asociado a la presencia de estrógenos de forma fisiológica, sin embargo, resulta paradójico que a pesar de haberse realizado estudios clínicos a escala global no se ha logrado determinar una disminución del riesgo en mujeres que usan terapia de reemplazo hormonal, incluso llegando a aumentar la prevalencia de estos desenlaces en muchas pacientes $^{4}$. Aunque sí se ha visto discreta disminución de la prevalencia de diabetes mellitus tipo 2 con esta terapia por mecanismos aún desconocidos ${ }^{5}$. Aparte, la forma de presentación de la enfermedad coronaria aguda por lo general es con síntomas autonómicos más que simplemente el dolor torácico que se presenta con mayor frecuencia en los hombres ${ }^{6}$. Es por esto que en esta revisión se pretende dar una nueva visión al quehacer diario de los médicos de atención primaria, quienes son los encargados en muchos casos de detectar de manera temprana y dirigir durante largos periodos de tiempo las terapias de prevención de eventos cardiovasculares mayores con base en la información de mayor impacto y específica para las patologías más asociadas al riesgo cardiovascular en las mujeres.

\section{Métodos}

Se realizó una búsqueda en PubMed y Google Scholar, en la primera con los términos MeSH "woman and diabetes mellitus not pregnancy" encontrando 85 resultados, "cardiovascular risk and woman" encontrando 132 resultados, y en Google Scholar se buscó enfermedad cardiovascular en mujeres diabéticas. Estos resultados se depuraron de acuerdo con el criterio de los autores y se obtuvieron algunas referencias cruzadas.

\section{Diferencias fisiopatológicas}

Se sabe que la pared ventricular izquierda es más delgada y contiene menos volumen en las mujeres al ajustarse al área corporal con respecto a los hombres, aunque posee mayor presión y fracción de eyección debido posiblemente a un volumen de final de sístole reducido. La fracción de eyección más elevada puede explicar la mayor prevalencia de insuficiencia cardiaca con fracción de eyección preservada en mujeres que en hombres. Además, las mujeres con fracción de eyección reducida tienden a requerir manejos más agresivos. Dentro de los factores de riesgo cardiovascular, se sabe que el intervalo QT es más ancho en mujeres, lo cual promueve una afectación de la repolarización ventricular'.

Los estrógenos, como hormonas predominantes en mujeres, poseen efectos fisiológicos cardiovasculares protectores como el aumento de la producción de óxido nítrico en células endoteliales (resultando en disminución de la contractilidad vascular y la ateroesclerosis), músculo liso, células miocárdicas y plaquetas (lo que resulta en menor agregación plaquetaria), inhibición del crecimiento y remodelación de las células miocárdicas y del músculo liso vascular, lo cual reduce a su vez la aterogénesis, aumenta la proliferación endotelial, la angiogénesis y reduce la constricción vascular. Los estrógenos también ejercen efectos sobre genes involucrados en el metabolismo cardiaco como la lipoproteína lipasa, la prostaglandina 2 sintasa y el coactivador 1 alfa del receptor activador de la proliferación de peroxisoma gamma. La poliol-aldosa reductasa y los estrógenos parecen estar implicados en el aumento del riesgo cardiovascular por el aumento de la producción de derivados glucados que están directamente implicados en las complicaciones del síndrome metabólico. Las corrientes de potasio de los cardiomiocitos inhibidos por la angiotensina II, al aumentar la duración del potencial de acción conducen a la insuficiencia cardiaca, sin embargo, este efecto no se presenta en mujeres con niveles normales de estrógenos, y a su vez, se ha asociado a polimorfismos en genes que regulan la expresión de la enzima convertidora de angiotensina. La enzima óxido nítrico sintasa, cuya alteración se asocia a disfunción endotelial y complicaciones cardiovasculares mayores, está directamente regulada hacia arriba por los estrógenos, lo cual permite mayor vasodilatación en mujeres y por lo tanto una propuesta de mecanismo de mejor respuesta a estímulos vasoconstrictores y proaterogénicos en mujeres premenopáusicas?. A pesar de que se han realizado 
estudios comparando los niveles de estrógenos y andrógenos en sangre con los de glucosa y su relación con el desarrollo de diabetes mellitus tipo 2, no se han encontrado asociaciones significativas, lo que implica que este no es un factor de peso para determinar el riesgo de eventos cardiovasculares ${ }^{8}$.

De los receptores de estrógenos, se conocen dos tipos, alfa y beta, los cuales se han encontrado en células endoteliales y en cardiomiocitos, y por medio de su activación se han evidenciado efectos antiapoptósicos, antihipertróficos y antiateroescleróticos. No obstante, aún quedan por esclarecer específicamente las combinaciones de receptores y hormonas que determinan la disminución del riesgo cardiovascular. Se han observado algunos polimorfismos de un solo nucleótido en diferentes poblaciones asociados a diferentes procesos biológicos involucrados en el riesgo cardiovascular, sin embargo la información es controvertida y muy poco estudiada para involucrar estos polimorfismos en decisiones terapéuticas ${ }^{9}$.

En cuanto a algunas hormonas, se conoce que la leptina actúa en el perfil de resistencia a la insulina, obesidad y diabetes, independientemente de la adiposidad. Los niveles de adiponectina son mayores en mujeres que en hombres, son inversamente proporcionales a la adiposidad visceral y su función está regulada por otras hormonas como la testosterona, prolactina, glucocorticoides y hormona de crecimiento. Curiosamente, el nivel de adiponectina se encuentra disminuido en mujeres posmenopáusicas, pero no se ha encontrado relación con los estrógenos como explicación para este evento. También se ha visto la acción del estradiol sobre citocinas proinflamatorias como el factor nuclear kappa $\mathrm{B}$, la proteína $\mathrm{C}$ reactiva y la resistina, siendo esta última relacionada con el nivel de triglicéridos, circunferencia de cintura, presión arterial sistólica, ratio apolipoproteína (APO) A1:APOB e inversamente relacionado a los niveles sanguíneos de lipoproteínas de alta densidad y APOA1, lo cual es un hallazgo específico en las mujeres ${ }^{4}$. Las mujeres poseen menor cantidad de ácidos grasos no esterificados circulantes y más densidad de apolipoproteina A1 (APO1) en sus lipoproteínas de alta densidad y menor tamaño en las lipoproteínas de baja densidad, lo que lleva a un perfil lipídico menos aterogénico en las mujeres que en los hombres. Esto es en parte explicado por la cantidad de enzimas lipoproteína lipasas $y$ de triglicéridos ${ }^{10}$. Además, los estrógenos contribuyen directamente a la reparación tisular, angiogénesis, aceleran la endotelización, también contribuyen a regular la respuesta inmunitaria innata y adaptativa, contribuyen al control del tono vascular y el perfil lipídico y según datos recientes, los receptores de estrógenos se expresan en células como monocito y macrófagos, y mediante estudios in vitro se ha encontrado que las alteraciones de estos receptores se relacionan con mayores efectos ateroescleróticos, obesidad y resistencia a la insulina ${ }^{7}$.

Los pacientes con diabetes mellitus tipo 2 poseen una función plaquetaria aumentada al igual que su adhesión, alteración en la coagulación por afectación de los factores antitrombóticos, lo cual induce un estado procoagulante, también hay una alteración de la matriz endotelial, la cual resulta en disminución de la reparación, menor respuesta al óxido nítrico y esto lleva posteriormente a la apoptosis de las células endoteliales ${ }^{11}$. Las mujeres con síndrome metabólico tienden a presentar menor volumen plaquetario, mayor agregación y densidad de receptores plaquetarios para unirse a fibrinógenos, lo cual a su vez va de la mano con el hecho de que es más común que las mujeres a quienes se administra antiagregantes pueden presentar más predisposición a sangrado gástrico sin mejorar el perfil de prevención primaria de eventos cardiovasculares ${ }^{12}$.

\section{Factores de riesgo cardiovascular en las mujeres}

En el estudio EUROASPIRE IV se encontró que las mujeres tienden a presentar tres o más factores de riesgo cardiovascular que los hombres, específicamente, las mujeres son menos conscientes de su obesidad central y hacen menos actividad física. La diferencia entre sexos se equiparó en cuanto al riesgo con el mayor nivel educativo y menor edad en mujeres ${ }^{13}$. Asturias fue un estudio observacional de cohortes de participantes entre 30 y 75 años, el cual buscaba encontrar diferencias entre sexo y presencia diabetes mellitus tipo II al evaluar desenlaces cardiovasculares y mortalidad, concluyendo que el riesgo de morir por algún ECM es tres veces mayor entre mujeres que tienen diabetes mellitus tipo II con respecto a las mujeres que no la tienen ${ }^{14}$. En un seguimiento de 13 años a 835 diabéticos y 1,296 no diabéticos, entre 45 y 64 años, se evidenció una alta prevalencia de desenlaces cardiovasculares mayores en mujeres diabéticas asociada a presión arterial elevada y dislipidemia aterogénica $^{15}$. En Cuba se determinó que las mujeres con edades entre 40 y 59 años con diabetes tienen aumento del riesgo cardiovascular con la menopausia y con el tiempo de evolución de esta ${ }^{16}$. 
Dentro de los factores que hacen parte del síndrome metabólico, la presión de pulso, índice cintura: cadera y el perfil lipídico analizado bajo transformación logarítmica se asociaron a peor perfil del riesgo cardiovascular en mujeres diabéticas ${ }^{17}$. En un análisis de datos de 5,243 personas del estudio Framingham entre $35 \mathrm{y}$ 74 años, se determinó que el riesgo de muerte por enfermedad coronaria para los hombres es mayor cuando han sufrido un evento coronario previo que cuando tienen diabetes, siendo este el caso contrario al de las mujeres. Además, al hacer un análisis de interacción diabetes-sexo, el riesgo relativo de mortalidad por enfermedad coronaria para las mujeres diabéticas fue de 2.31 (intervalo de confianza del 95\% [IC95\%]: 1.25-4.23) ${ }^{18}$. Por otra parte, se ha descrito que las mujeres tienden a seguir en menor medida las medidas no farmacológicas como la dieta mediterránea con respecto a los hombres, y los hombres presentan como factor de riesgo independiente el fumar cigarrillos y la presión arterial para el desarrollo de eventos cardiovasculares a 10 años ${ }^{19}$.

En el estudio Maastricht, que incluyó 1,654 mujeres, se evidenció que las mujeres en promedio pasan hasta 10 años y los hombres 8 años con diagnóstico de prediabetes antes de presentar diabetes tipo 2, además las mujeres con estado glucémico normal tuvieron mejor perfil cardiovascular que los hombres, aunque los niveles de hemoglobina glucosilada se asociaron a peor estado metabólico en mujeres sin diabetes mellitus, en tanto que las mujeres con diabetes mellitus tuvieron peor perfil cardiometabólico que los hombres $^{20}$. En un metaanálisis que incluyó 49 estudios, se comparó el efecto de la diabetes mellitus en el riesgo cardiovascular, cáncer, mortalidad por enfermedades respiratorias e infecciones entre hombres y mujeres, encontrando una incidencia un $30 \%$ mayor de muerte por causa cardiovascular en mujeres con diabetes que en hombres (reducción de riesgo relativo (RRR): 1.30; IC 95\%: 1.13-1.49), además las mujeres con diabetes presentaron un $58 \%$ más de riesgo para mortalidad por enfermedad coronaria que los hombres (RRR: 1.58; IC 95\%: 1.32-1.90), pero solo el $8 \%$ de mortalidad por enfermedad cerebrovascular (RRR: 1.08; IC 95\%: $1.01-1.15)^{21}$.

\section{Condiciones de las mujeres y su relación con enfermedad cardiovascular}

El embarazo aumenta el riesgo aterogénico considerablemente al manifestarse como diabetes gestacional y preeclampsia, asociado al aumento de la dislipidemia y la resistencia a la insulina. Incluso se ha descrito a la preeclampsia como la primera manifestación del síndrome metabólico en muchas mujeres. Posterior al segundo embarazo, se aumenta el riesgo de enfermedad coronaria aguda hasta en un $30 \%$, cuya causa se cree que es la detención del estímulo protector de los estrógenos durante el embarazo y el aumento de la resistencia a la insulina ${ }^{22}$.

Las mujeres sin enfermedad cardiovascular previa que presentaron enfermedades mediadas por alteraciones de la placenta presentaron un riesgo dos veces mayor para el desarrollo de enfermedades cardiovasculares, con preeclampsia riesgo relativo (RR): 4.19 [IC 95\%: $2.09-8 .^{38}$ para insuficiencia cardiaca y a preeclampsia para el desarrollo de hipertensión crónica hazard ratio [HR]: 5.6 (IC 95\%: 5.1-6.3) ${ }^{23}$. Las mujeres que padecen diabetes gestacional tienen un riesgo de ocho veces mayor para desarrollar diabetes mellitus tipo 2 comparado con mujeres con niveles de glucemia normales. También se ha asociado con mayor incidencia de eventos cardiovasculares posteriores con una oportunidad del $63 \%$ y un aumento del riesgo absoluto del $2.8 \%$. En mujeres con diabetes gestacional mediante un seguimiento posterior a la gestación y comparando mujeres que desarrollaron diabetes mellitus tipo 2 y mujeres que no RR: 1.56 (IC 95\%: 1.04-2.32), con lo cual se ha concluido que la diabetes gestacional es un factor de riesgo independiente para el desarrollo de enfermedades cardiovasculares ${ }^{24}$.

En cuanto al parto pretérmino, especialmente los que ocurren antes de la semana 32 de gestación, también se han descrito como un factor de riesgo independiente para el desarrollo de enfermedades cardiovasculares. Las mujeres con embarazo pretérmino se han asociado a un aumento en la incidencia de hipercolesterolemia, diabetes mellitus tipo 2, hipertensión crónica, y en seguimiento de hasta de 10 años son más susceptibles a desarrollar ateroesclerosis subclínica. Se sabe que esta es solo el $25 \%$ de la relación del parto pretérmino con la enfermedad cardiovascular, se conocen algunos mecanismos posiblemente asociados a estos eventos aunque aún los datos son limitados. Hay evidencia que respalda la asociación entre la gestación de bajo peso y el riesgo cardiovascular, no obstante, se cree que es debido a defectos de la placenta, ya que las mujeres con gestaciones de bajo peso poseen menor capacidad de adaptación a los cambios hemodinámicos del embarazo ${ }^{25}$.

La insuficiencia ovárica involucra hipergonadismo, hipoestrogenismo y menopausia antes de los 45 años de edad se relacionan con un aumento del riesgo 
cardiovascular (RR: 1.50; IC 95\%: 1.28-1.76); esto concuerda con los datos mencionados anteriormente sobre los efectos benéficos de los estrógenos en la salud endotelial, el perfil metabólico y que al presentarse la menopausia se pierde este efecto protector ${ }^{25}$. Por otro lado, la lactancia materna tiene potenciales efectos benéficos en disminución del riesgo cardiovascular por los efectos metabólicos que la rodean como aumentar la sensibilidad a la insulina, mejorar el perfil lipídico y disminuir la masa grasa acumulada durante la gestación ${ }^{25}$.

\section{Panorama de la prevención del riesgo cardiovascular en las mujeres}

En las mujeres posmenopáusicas es conocido que hay una igualdad relativa de incidencia en el riesgo de desarrollo de la enfermedad cardiovascular respecto a los hombres por la pérdida del factor protector que suponen los estrógenos en ellas, sin embargo, es interesante analizar que en las mujeres la presentación clínica de la enfermedad coronaria aguda tiende a ser diferente y más aún en las mujeres diabéticas, por lo que en este grupo podría suponer un reto clínico en muchas ocasiones y además está comprobado que las mujeres tienden a no ser tan conscientes de los síntomas de un infarto al momento de presentarlos ${ }^{26-30}$. Hay una tendencia mundial a no identificar a tiempo los factores de riesgo cardiovascular específicos en mujeres, menospreciar el mayor detrimento de la enfermedad cardiovascular en mujeres diabéticas, un inicio en general más tardío de terapias con estatinas, aspirina y manejo no farmacológico de factores de riesgo modificables ${ }^{31-33}$. Esto ha sido explicado debido a la falta identificación por parte del personal médico de primera línea e incluso algunos especialistas de los factores de riesgo cardiovascular en mujeres más que en hombres, aun cuando estos están presentes ${ }^{34}$. Por ejemplo, ya se conoce que la preeclampsia (RR: 2.50; IC 95\%: 1.43-4.37), diabetes gestacional, enfermedades autoinmunes y la hipertensión gestacional, son factores de riesgo específicos en mujeres para el desarrollo de enfermedades cardiovasculares, sin embargo, hasta el momento no se han tomado decisiones en las guías de atención locales y mundiales para aminorar estos efectos $^{25,35-38}$.

\section{Conclusiones}

Son muchos los factores genéticos y modificables asociados al riesgo cardiovascular en las mujeres $y$ especialmente en aquellas que son diabéticas. Es sabido que estos factores de riesgo son mayoritariamente modificables con estilos de vida saludables y cada vez es más necesario generar espacios de reflexión en la población para adherirse a estilos de vida saludables y a los médicos de atención primaria para tener en cuenta las particularidades de las mujeres en el desarrollo de la enfermedad cardiovascular y tomar decisiones terapéuticas oportunas, como en el caso de aquellas que han tenido enfermedades durante la gestación o consumen anticonceptivos orales de larga data antes de que estas patologías progresen hacia desenlaces cardiovasculares mayores en sus pacientes.

\section{Financiamiento}

La realización del presente artículo fue autofinanciada.

\section{Conflicto de intereses}

Los autores declaran que no existe ningún conflicto de intereses

\section{Responsabilidades éticas}

Protección de personas y animales. Los autores declaran que para esta investigación no se han realizado experimentos en seres humanos ni en animales.

Confidencialidad de los datos. Los autores declaran que han seguido los protocolos de su centro de trabajo sobre la publicación de datos de pacientes.

Derecho a la privacidad y consentimiento informado. Los autores declaran que en este artículo no aparecen datos de pacientes.

\section{Bibliografía}

1. Garcia M, Mulvagh SL, Merz CNB, Buring JE, Manson JE. Cardiovascular disease in women: Clinical perspectives. Circ Res. 2016;118(8):1273-93.

2. Sattar N. Type 2 diabetes-related sex differences in cardiovascular risk: reasons, ramifications, and clinical realities. Eur Heart J. 2020;41(13):1354-6.

3. Nadal JF, Gutiérrez PC. ¿Es necesario el tratamiento integral de la diabetes mellitus tipo 2 y los factores de riesgo cardiovascular? Med Clin. 2013;141:7-13.

4. Ren J, Kelley RO. Cardiac health in women with metabolic syndrome: clinical aspects and pathophysiology. Obesity (Silver Spring). 2009;17(6):1114-23.

5. Mauvais-Jarvis F, Manson JE, Stevenson JC, Fonseca VA. Menopausal hormone therapy and type 2 diabetes prevention: Evidence, mechanis$\mathrm{ms}$, and clinical implications. Endocr Rev. 2017;38(3):173-88.

6. García M. Factores de riesgo cardiovascular desde la perspectiva de sexo y género. Rev Colomb Cardiol. 2018;25:8-12.

7. Trenti A, Tedesco S, Boscaro C, Trevisi L, Bolego C, Cignarella A. Estrogen, angiogenesis, immunity and cell metabolism: Solving the puzzle. Int J Mol Sci. 2018:19(3).

8. Kim C, Halter JB. Endogenous sex hormones, metabolic syndrome, and diabetes in men and women. Curr Cardiol Rep. 2014;16(4):467.

9. Morselli E, Santos RS, Criollo A, Nelson MD, Palmer BF, Clegg DJ. The 
effects of oestrogens and their receptors on cardiometabolic health. Nat Rev Endocrinol. 2017;13(6):352-64.

10. Colhoun $\mathrm{H}$. Coronary heart disease in women: why the disproportionate risk? Curr Diab Rep. 2006;6(1):22-8.

11. Kaseta JR, Skafar DF, Ram JL, Jacober SJ, Sowers JR. Cardiovascular disease in the diabetic woman. J Clin Endocrinol Metab. 1999;84(6):1835-8.

12. Gasperi V, Catani MV, Savini I. Platelet responses in cardiovascular disease: Sex-related differences in nutritional and pharmacological interventions. Cardiovasc Ther. 2020;2020:2342837.

13. De Smedt D, De Bacquer D, De Sutter J, Dallongeville J, Gevaert S, De Backer $\mathrm{G}$, et al. The gender gap in risk factor control: Effects of age and education on the control of cardiovascular risk factors in male and female coronary patients. The EUROASPIRE IV study by the European Society of Cardiology. Int J Cardiol. 2016;209:284-90.

14. Ares Blanco J, Valdés Hernández $S$, Botas $P$, Rodríguez-Rodero $S$, Morales Sánchez P, Díaz Naya L, et al. Diferencias de género en la mortalidad de personas con diabetes tipo 2: Estudio Asturias 2018. Gac Sanit. 201934(5):442-8.

15. Juutilainen A, Kortelainen S, Lehto S, Rönnemaa T, Pyörälä K, Laakso M Gender difference in the impact of type 2 diabetes on coronary heart disease risk. Diabetes Care. 2004;27(12):2898-904.

16. Valdés Ramos E, Castillo Oliva Y, Valdés Bencosme E. Estimación del riesgo cardiovascular global en mujeres diabéticas de edad mediana. Rev Cuba Endocrinol. 2017;28(3):1-10.

17. Wakabayashi I. Gender differences in cardiovascular risk factors in patients with coronary artery disease and those with type 2 diabetes. J Thorac Dis. 2017;9(5):E503-6.

18. Natarajan S, Liao Y, Cao G, Lipsitz SR, McGee DL. Sex differences in risk for coronary heart disease mortality associated with diabetes and established coronary heart disease. Arch Intern Med. 2003;163(14):1735-40.

19. Kouvari M, Panagiotakos DB, Chrysohoou C, Georgousopoulou E, Notara V, Tousoulis D, et al. Gender-specific, lifestyle-related factors and 10-year cardiovascular disease risk; the ATTICA and GREECS Cohort Studies. Curr Vasc Pharmacol. 2019;17(4):401-10.

20. de Ritter R, Sep SJS, van der Kallen CJH, Schram MT, Koster A, Kroon AA, et al. Adverse differences in cardiometabolic risk factor levels between individuals with pre-diabetes and normal glucose metabolism are more pronounced in women than in men: the Maastricht Study. BMJ Open Diabetes Res Care. 2019:7(1):e000787.

21. Wang Y, O'Neil A, Jiao Y, Wang L, Huang J, Lan Y, et al. Sex differences in the association between diabetes and risk of cardiovascular disease, cancer, and all-cause and cause-specific mortality: a systematic review and meta-analysis of 5,162,654 participants. BMC Med. 2019;17(1):136.

22. Araujo LF, de Matos Soeiro A, Fernandes JL, Pesaro AE, Serrano CV. Coronary artery disease in women: a review on prevention, pathophysiology, diagnosis, and treatment. Vasc Health Risk Manag. 2006;2(4):465-75.

23. Wu P, Haththotuwa R, Kwok CS, Babu A, Kotronias RA, Rushton C, et al. Preeclampsia and future cardiovascular health: A systematic review and meta-analysis. Circ Cardiovasc Qual Outcomes. 2017;10(2):e003497.
24. Kramer CK, Campbell S, Retnakaran R. Gestational diabetes and the risk of cardiovascular disease in women: a systematic review and meta-analysis. Diabetologia. 2019;62(6):905-14.

25. Agarwala A, Michos ED, Samad Z, Ballantyne CM, Virani SS. The use of sex-specific factors in the assessment of women's cardiovascular risk. Circulation. 2020;141(7):592-9.

26. Stephen SA, Darney BG, Rosenfeld AG. Symptoms of acute coronary syndrome in women with diabetes: an integrative review of the literature. Heart Lung J Crit Care. 2008;37(3):179-89.

27. Patel $H$, Rosengren $A$, Ekman I. Symptoms in acute coronary syndromes: does sex make a difference? Am Heart J. 2004;148(1):27-33.

28. Berman N, Jones MM, De Coster DA. «Just like a normal pain», what do people with diabetes mellitus experience when having a myocardial infarction: a qualitative study recruited from UK hospitals. BMJ Open. 2017;7(9):e015736.

29. Lichtman JH, Leifheit-Limson EC, Watanabe E, Allen NB, Garavalia B, Garavalia LS, et al. Symptom recognition and healthcare experiences of young women with acute myocardial infarction. Circ Cardiovasc Qual Outcomes. 2015;8(2 Suppl 1):S31-8.

30. van Oosterhout Roos EM, de Boer Annemarijn R, Maas Angela HEM, Rutten Frans H, Bots Michiel L, Peters Sanne AE. Sex differences in symptom presentation in acute coronary syndromes: A systematic review and metaワanalysis. J Am Heart Assoc. 2020;9(9):e014733.

31. Zhao M, Woodward M, Vaartjes I, Millett ERC, Klipstein-Grobusch K, Hyun K, et al. Sex differences in cardiovascular medication prescription in primary care: A systematic review and meta-analysis. J Am Heart Assoc. 2020;9(11):e014742.

32. Palau P, Bertomeu-González V, Sanchis J, Soler M, de la Espriella R, Domínguez $\mathrm{E}$, et al. Efecto pronóstico diferencial de la diabetes mellitus tipo 2 en mujeres y varones con insuficiencia cardiaca y fracción de eyección conservada. Rev Esp Cardiol. 2020;73(6):463-70.

33. Feng $A$, Peña $Y$, Li W. La cardiopatía isquémica en pacientes diabéticos y no diabéticos. Rev Habanera Cienc Médicas. 2017;16(2):217-28.

34. Daugherty SL, Blair IV, Havranek EP, Furniss A, Dickinson LM, Karimkhani $\mathrm{E}$, et al. Implicit gender bias and the use of cardiovascular tests among cardiologists. J Am Heart Assoc Cardiovasc Cerebrovasc Dis. 2017;6(12):e006872.

35. Goldstein KM, Zullig LL, Bastian LA, Bosworth HB. Statin adherence: Does gender matter? Curr Atheroscler Rep. 2016;18(11):63.

36. Brush JE, Krumholz HM, Greene EJ, Dreyer RP. Sex differences in symptom phenotypes among patients with acute myocardial infarction. Circ Cardiovasc Qual Outcomes. 2020;13(2):e005948.

37. Abuful A, Gidron Y, Henkin Y. Physicians' attitudes toward preventive therapy for coronary artery disease: is there a gender bias? Clin Cardiol. 2005;28(8):389-93.

38. Daugherty SL, Blair IV, Havranek EP, Furniss A, Dickinson LM, Karimkhani $E$, et al. Implicit gender bias and the use of cardiovascular tests among cardiologists. J Am Heart Assoc. 2017;6(12). 